\section{ALL emerges from relapse}

\section{By Lauren Martz, Staff Writer}

Separate teams led by researchers from New York University and Columbia University have identified mutations in $5^{\prime}$-nucleotidase cytosolic II that predict relapse, drug resistance and poor prognosis in acute lymphoblastic leukemia. ${ }^{1,2}$ Inhibiting the enzyme could improve disease outcomes and sensitize some relapsed patients to marketed acute lymphoblastic leukemia therapies.

Current treatment regimens for ALL involve first-line chemotherapy followed by maintenance therapy that includes the purine analogs 6-mercaptopurine (6-MP) and 6-tioguanine (6-TG).

Although the treatments are effective for many patients, about $10 \%-20 \%$ of pediatric patients and up to $50 \%$ of adult patients relapse.

"If one were to target
NT5C2, the potential
danger would lie in
inhibiting the normal
function of NT5C2 in purine
metabolism."
- Cancer Research UK

"If one were to target NT5C2, the potential danger would lie in inhibiting the norma function of NT5C2 in purine - Josef Vormoor, the cells resistant to apoptosis by 6-MP and 6-TG compared with expression of the wild-type gene.

The paper also included researchers from St. Jude Children's Research Hospital, the Memorial Sloan-Kettering Cancer Center, Weill Cornell Medical College, the University of Colorado Denver School of Medicine and Children's Hospital Colorado.

In a paper published in Nature Medicine, a Columbia University team led by Adolfo Ferrando published similar findings for patients with a highly aggressive ALL subtype-T cell ALL. Ferrando is an associate professor of pathology and pediatrics at Columbia's Institute for Cancer Genetics.

Ferrando's group used matched whole-exome sequencing on samples from five patients with T-ALL at diagnosis, remission and relapse and identified a relapse-associated mutation in NT5C2 in one of the patients.

The group then studied a panel of 98 relapsed T-ALL and 35 relapsed B precursor ALL samples. NT5C2 was mutated in 1 additional patient with B-ALL and 19 additional patients with T-ALL. The mutations were absent in matched samples from the time of diagnosis.

In a $5^{\prime}$-nucleotidase assay, the mutant enzymes had up to 48 -fold greater activity than wild-type enzyme. In T-ALL cells, vector-mediated expression of the mutant genes increased cell viability with increasing concentrations of 6-MP or 6-TG compared The relapsed disease often is resistant to drugs and has a cure rate of less than $40 \% .^{3}$

The two teams conducted genetic studies on patient samples to find the underlying mechanisms of ALL relapse. Both converged on overlapping sets of gain-of-function mutations in $5^{\prime}$-nucleotidase cytosolic II (NT5C2), which were associated with disease relapse and could be causing drug resistance.

In a paper published in Nature Genetics, William Carroll and colleagues at NYU sequenced RNA samples from pediatric patients with B lymphoblastic leukemia, the most common form of ALL. Carroll is a professor of pediatrics and director of the NYU Cancer Institute.

The team found 20 missense mutations in samples from 10 relapse patients that were absent in matched samples from patients at diagnosis or during remission, including two mutations in the coding region of $N T 5 C 2$.

In a follow-up analysis of samples from another 61 relapse patients, full exon sequencing of NT5C2 identified additional mutations in 5 more patient samples, suggesting the gene is mutated about $10 \%$ of the time. Moreover, every patient who expressed the NT5C2 mutations relapsed within 36 months of initial diagnosis.

Mechanistic studies showed that expression of the NT5C2 mutants in Escherichia coli led to greater 5 '-nucleotidase activity than expression of wild-type NT5C2. In cultured B lymphoblastic leukemia cells, lentivirus-mediated expression of mutant NT5C2 rendered with expression of wild-type NT5C2 or an empty vector control.

The paper also included researchers from the Albert Einstein College of Medicine of Yeshiva University, Charité-University Hospital Berlin, Cancer Institute Padua, the Technion-Israel Institute of Technology, Memorial Sloan-Kettering, the Shaare Zedek Medical Center and the University of Padua.

Carroll, corresponding author of the Nature Genetics paper, told SciBX that his team was encouraged by the results of the Nature Medicine paper "because we found the relapse-associated mutation in the B cell subset of ALL, and they have found it in the other form, T-ALL. These are different biological subtypes, and it is interesting that regardless of the type of cancer, common treatments may lead to the emergence of the same resistance mechanisms due to the selective pressure. This is a form of convergent evolution. To us, this means that other cancers, particularly adult forms of ALL, may also be affected by the same resistance mechanisms."

\section{NT5C2 inhibitors}

Both teams told SciBX that their next steps included designing NT5C2 inhibitors. The molecules likely would be used to resensitize resistant ALL to standard therapies like 6-MP and 6-TG.

"If one were to target NT5C2, the potential danger would lie in inhibiting the normal function of NT5C2 in purine metabolism," noted Josef Vormoor, a Cancer Research UK childhood leukemia expert and professor of child health at Newcastle University. 
Carroll and colleagues hope to avoid that problem by designing inhibitors that selectively bind the mutant form of the enzyme.

"Our ultimate goal is to design inhibitors specific for the mutant forms of the enzymes to spare the wild-type enzyme function. We have not yet seen if this is possible, and designing selective compounds has been challenging, but these studies are underway," said Carroll.

"The cellular function of NT5C2 is to clear purine nucleoside intermediates out of the cell in conditions of high energy. At this point, it is unclear what would be the systemic effects of NT5C2 inhibition," added corresponding author Ferrando.

In addition to therapeutic discovery, Ferrando told SciBX that his team is developing diagnostic assays to identify NT5C2 mutations as biomarkers of prognosis and drug resistance.

He noted that there are alternative therapeutics for patients with leukemia who do not respond to 6-MP or other purine drugs, so it would be beneficial to be able to predict response.

For example, Ferrando's team showed that nelarabine, an approved drug in the purine pathway, was active against NT5C2-mutated cancer cell lines. He added that other antileukemic drugs with different mechanisms of action including glucocorticoids and methotrexate also are active in NT5C2-mutated leukemias.

GlaxoSmithKline plc markets Arranon nelarabine to treat ALL and lymphoma.

Carroll said a key goal for his team is to prevent global resistance before it begins. "Once frank relapse occurs, other mutations have likely emerged, causing pan-resistance. The chances that restoring sensitivity to purine analogs will be sufficient to cure the cancer alone are reduced," he said. "We are now looking into whether we can use next-generation sequencing of patient samples throughout disease remission to see whether we can catch these mutations early as they emerge to alter the therapeutic regimen before full relapse occurs."
Carroll said NYU has filed a patent application covering the mutations and that the IP is available for licensing.

Ferrando told SciBX that Columbia has filed a patent application for the NT5C2 mutations for diagnostic and therapeutic applications. The IP is available for licensing.

Martz, L. SciBX 6(9); doi:10.1038/scibx.2013.208

Published online March 7, 2013

\section{REFERENCES}

1. Meyer, J.A. et al. Nat. Genet.; published online Feb. 3, 2013; doi:10.1038/ng.2558

Contact: William L. Carroll, NYU Cancer Institute, New York, N.Y. e-mail: william.carroll@nyumc.org

2. Tzoneva, G. et al. Nat. Med.; published online Feb. 3, 2013; doi:10.1038/nm.3078

Contact: Adolfo Ferrando, Columbia University, New York, N.Y. e-mail: af2196@columbia.edu

3. Pui, C.-H. \& Evans, W.E. et al. N. Engl. J. Med. 354, 166-178 (2006)

\section{COMPANIES AND INSTITUTIONS MENTIONED}

Albert Einstein College of Medicine of Yeshiva University, New York, N.Y.

Cancer Institute Padua, Padua, Italy

Cancer Research UK, London, U.K.

Charité-University Hospital Berlin, Berlin, Germany

Children's Hospital Colorado, Aurora, Colo.

Columbia University, New York, N.Y.

GlaxoSmithKline plc (LSE:GSK; NYSE:GSK), London, U.K. Memorial Sloan-Kettering Cancer Center, New York, N.Y. Newcastle University, Newcastle upon Tyne, U.K.

New York University, New York, N.Y.

Shaare Zedek Medical Center, Jerusalem, Israel

St. Jude Children's Research Hospital, Memphis, Tenn.

Technion-Israel Institute of Technology, Haifa, Israel

University of Colorado Denver School of Medicine, Aurora, Colo.

University of Padua, Padua, Italy

Weill Cornell Medical College, New York, N.Y. 